Classification

Physics Abstracts

$71.55 \mathrm{~F}-78.55 \mathrm{D}$

\title{
Binding to a pair of marginal potentials : a test for the stability of $\mathbf{N}^{-}$in $\mathbf{G a P}$
}

\author{
C. Benoît à la Guillaume \\ Groupe de Physique des Solides de l'E.N.S. (*), Tour 23, Université de Paris VII, 2 Place Jussieu, \\ 75251 Paris Cedex 05, France
}

(Reçu le 17 septembre 1982, révisé le 12 novembre, acceptéle 16 novembre 1982)

\begin{abstract}
Résumé. - On montre que le comportement des paires distantes $\mathrm{NN}^{-}$fournit un test de stabilité de $\mathrm{N}^{-}$. On propose une méthode améliorée de calcul de l'énergie de $\mathrm{NN}^{-}$, ainsi que du comportement asymptotique à grande distance $R$ pour un potentiel près de la limite de liaison. On démontre que l'énergie de liaison à une paire de potentiels " marginaux » est une fonction universelle de $R$, et indépendante de la nature du potentiel. On conclut que $\mathrm{N}^{-}$n'est pas stable dans $\mathrm{GaP}$.
\end{abstract}

\begin{abstract}
It is shown that a test for the stability of $\mathrm{N}^{-}$can be found from the behaviour of pairs $\mathrm{NN}_{i}^{-}$at large separation. An improved method of calculation of the energy of $\mathrm{NN}_{i}^{-}$is presented and its asymptotic behaviour at large pair separation $R$ is obtained for potential near the limit of vanishing binding. It is shown that the binding energy to a pair of « marginal » potentials is a universal function of $R$, independent of the nature of the potential. It is concluded that $\mathrm{N}^{-}$is unstable in $\mathrm{GaP}$.
\end{abstract}

1. Introduction. - $\mathbf{N}$ substituted to $\mathrm{P}$ in $\mathrm{GaP}$ is the most thoroughly studied shallow isoelectronic centre [1]. $\mathrm{N}$ being more electronegative than $\mathrm{P}$ causes an attractive short-range potential for the electron [2] but one does not know if $\mathrm{N}^{-}$is stable or not in $\mathrm{GaP}$. In this paper, we propose to find a test of stability of $\mathrm{N}^{-}$from the study of $\mathrm{NN}_{i}^{-}$, an electron bound to a pair of nitrogen; good experimental data about $\mathrm{NN}_{i}^{-}$have been obtained by Cohen and Sturge [3] from excitation spectroscopy of the luminescence of excitons bound to pairs. Here, we propose an improved method, yet very simple, to compute the ground state of $\mathrm{NN}_{i}^{-}$as a function of the pair separation and the depth of the localized isoelectronic potential. In addition, we found an asymptotic law for the binding energy $E$ to a pair of «marginal " potentials at large pair separation $R$ : $E R^{2}=$ const., independent of the nature of the potential. A simple test for $\mathrm{N}^{-}$stability is derived.

2. Theoretical calculation of $\mathbf{N N}_{i}^{-}$ground state - Let $V(r)$ the isoelectronic potential spherically symmetric. It is assumed of constant shape but with a prefactor $(1+\varepsilon)$. $V(r)$ is chosen such that being short-range, the binding energy of the electron is just vanishing : this is our definition of a "marginal» potential. Actual potential $(1+\varepsilon) V(r)$ has a ground state of energy $E(\varepsilon)$ for $\varepsilon>0$.

(*) Laboratoire associé au CNRS. 
For a pair at separation $R$, we use a variational method, with a trial wave function of the bonding type :

$$
\Psi=\left(\phi_{1 \eta}+\phi_{2 \eta}\right) / \sqrt{2}
$$

$\phi_{i \eta}$, centred on impurity $i(i=1,2)$ is the solution for a potential $(1+\eta) V(r)$ :

$$
[K+(1+\eta) V(r)-E(\eta)] \phi_{\eta}=0,
$$

where $\eta$ is the variational parameter.

This trial wave function is exact in the two limiting cases $R=0$ where $1+\eta=2(1+\varepsilon)$ give the solution, and $R \rightarrow \infty$ where $\eta=\varepsilon$. Thus it is expected to be quite good at any value of $R$; it gives much better results then the choice $\phi \sim \exp (-r / a)$ as used in [4]. The variational energy $E_{1}$ is :

$$
E_{1}=E(\eta)+[(1+\varepsilon)(B+C)+(\varepsilon-\eta)(A+B)] /(1+D)
$$

where

$$
\begin{array}{ll}
A=\int \phi_{1 \eta} V_{1} \phi_{1 \eta} \mathrm{d}^{3} r & B=\int \phi_{1 \eta} V_{1} \phi_{2 \eta} \mathrm{d}^{3} r \\
C=\int \phi_{1 \eta} V_{2} \phi_{1 \eta} \mathrm{d}^{3} r & D=\int \phi_{1 \eta} \phi_{2 \eta} \mathrm{d}^{3} r .
\end{array}
$$

Numerical calculations as a function of $\varepsilon$ and $R$ have been performed in the case of a square well potential : $V(r)=\frac{-\Pi^{2}}{4 b^{2}}$ for $r<b, V(r)=0$ for $r>b$ ( $b$ is the radius of the localized potential). The results are shown in Section 4.

We find that the minimum $E_{1 \mathrm{~m}}$ of $E_{1}$ is obtained for a value $\eta=\eta_{\mathrm{m}}$ such that $E_{1 \mathrm{~m}} \sim E\left(\eta_{\mathrm{m}}\right)$. This is not surprising for the following reason : outside the two localized potentials, the ground state wave function of $\mathrm{NN}^{-}$is a linear combination of plane waves of energy $E_{0}$, where $E_{0}$ is the ground state energy. The ansatz (1) outside the two localized potentials is also a linear combination of plane waves of energy $E(\eta)$. If (1) is a good ansatz, then clearly one should have $E_{0}=E\left(\eta_{\mathrm{m}}\right)$.

3. Asymptotic behaviour. - We consider the case $\varepsilon=0, R \rightarrow \infty$. Since the ansatz (1) is exact at $R \rightarrow \infty$, we can use the " physical " argument of the end of Section 2 and replace the minimization of $E_{1}$, by $E_{1}=E(\eta)$ in equation (3), hence

$$
(1+\varepsilon)(B+C)+(\varepsilon-\eta)(A+B)=0 .
$$

Indeed, we show in the appendix that we can arrive at the same result by a direct (but more tedious) derivation from equation (3). We take $\hbar=m=1$.

To evaluate $A, B$ and $C$, we consider $f_{0}=r \phi_{0} ; f_{0}$ is solution of

$$
\frac{\mathrm{d}^{2} f_{0}}{\mathrm{~d} r^{2}}=V f_{0} \text {. }
$$

The normalization is chosen such that $f_{0}=1$ at $r \rightarrow \infty$. For a small $\eta, f_{\eta}$ is essentially equal to $f_{0}$ inside the potential, and like $\exp (-k r)$ outside the potential, where $k=\sqrt{-E(\eta)}$. To normalize 
$\phi_{\eta}$ as usually, the main contribution comes from the region outside the potential, introducing a factor $N^{2}=\frac{k}{2 \Pi}$. So we have

$$
\begin{array}{ll}
\phi_{\eta}=N f_{0} / r & \text { inside the potential, } \\
\phi_{\eta}=N \exp (-k r) / r & \text { outside the potential. }
\end{array}
$$

Then one obtains

$$
\begin{aligned}
& A=2 k I_{A} \\
& B=2 k I_{B} \exp (-k R) / R \\
& C=2 k I_{C} \exp (-2 k R) / R^{2},
\end{aligned}
$$

where

$$
I_{A}=\int f_{0}^{2} V \mathrm{~d} r \quad I_{B}=\int r f_{0} V \mathrm{~d} r
$$

and

$$
I_{C}=\int r^{2} V \mathrm{~d} r .
$$

$I_{A}^{-1}$ has the dimension of a length. It can be considered as the " effective " radius of the marginal potential (for a square well : $I_{A}^{-1}=-8 b / \Pi^{2}$ ).

$I_{B}$ can be computed using (5)

$$
\begin{aligned}
& I_{B}=\int r \frac{\mathrm{d}^{2} f_{0}}{\mathrm{~d} r^{2}} \mathrm{~d} r=\int \frac{\mathrm{d}}{\mathrm{d} r}\left(r \frac{\mathrm{d} f_{0}}{\mathrm{~d} r}-f_{0}\right) \mathrm{d} r=\left|r \frac{\mathrm{d} f_{0}}{\mathrm{~d} r}-f_{0}\right|_{0}^{\infty}, \\
& I_{B}=-1 \text { provided } r \frac{\mathrm{d} f_{0}}{\mathrm{~d} r}=0 \text { when } r \rightarrow \infty .
\end{aligned}
$$

This condition does not introduce any limitation on $V$ for the following reason : if when $r \rightarrow \infty$, $f_{0}=1-u r^{-s}$ then $r \frac{\mathrm{d} f_{0}}{\mathrm{~d} r} \sim r^{-s}$ and $V \sim r^{-(s+2)}, r \frac{\mathrm{d} f_{0}}{\mathrm{~d} r} \rightarrow 0$ if $s>0$, and so $V$ should decay faster than $r^{-2}$. This is a necessary condition for a marginal potential [5].

$I_{C}$ has the dimension of a length $\left(I_{C}=-\Pi^{2} b / 12\right.$ for a square well). The approximate way we have computed $C$ implies that $V$ should decay faster than $r^{-3}$.

For large $R$, (6) show that $A \gg B \gg C$. This comes from the power dependence in $R$, and not from the exponential term, because at the end it happens that $k R$ remains finite.

For $\varepsilon=0$, equation (4) reduces to

$$
B-\eta A=0 \text {. }
$$

Now, from the differentiation of equation (2) we get $A=\frac{\mathrm{d} E(\eta)}{\mathrm{d} \eta}$ hence, using $(7)$ and $E(\eta)=-k^{2}$,

$$
I_{A}=-\frac{\mathrm{d} k}{\mathrm{~d} \eta}, \text { then } k=-I_{A} \eta,
$$

since $k=0$ at $\eta=0$.

Then (8) becomes

$$
\exp (-k R)=k R .
$$


The numerical result is $k R=0.567$ or

$$
E=-0.322 / R^{2}
$$

Thus, the binding of a particle to a pair of «marginal» potentials is, at large pair separation, a universal function of $R$, independent of the nature of the potential.

For $\varepsilon$ small, (4) can be approximated as

$$
k R+\varepsilon I_{A} R=\exp (-k R) .
$$

This shows that the relevant parameter to measure a distance to the "marginal " case is $\varepsilon I_{A}$ (rather than $\varepsilon$ ). In particular, if $\varepsilon<0$ the distance $R_{0}$ at which the binding to a pair vanishes is

$$
R_{0}=1 /\left(\varepsilon I_{A}\right)
$$

4. Comparison with $\mathbf{N}$ in GaP. - Figure 1 gives the results of $\mathrm{NN}^{-}$ground state energy calculation for a square well potential, as a function of $R$ and for five values of $\varepsilon$ (from $-10 \%$ to $10 \%$ ). The asymptotic law (10) has also been drawn. On the same graph, we have plotted the experimental points coming from reference [3]. The only parameter of interest is the electron effective mass in GaP. Since we are interested in very shallow states, there is no ambiguity. We have taken $m^{*}=0.5$, which gives associated with $K=11$ an electron Rydberg of $55 \mathrm{meV}$ (the energy of the valley orbit splitted states of the donor). In reference [3], Cohen and Sturge measure the excitation spectrum of the luminescence of an exciton bound to a pair $\mathrm{NN}_{i}$. The pair separation is $R_{i}=d \sqrt{i / 2}$, where $d$ is the lattice constant $(\sim 5.4 \AA$ in GaP $)$. The excited states correspond to $n \mathrm{~S}$ orbitals of the hole wave function. The ionization limit, corresponding to $\mathrm{NN}_{i}^{-}$, is obtained by an extrapolation procedure, the binding energy of $\mathrm{NN}_{i}^{-}$is $E_{\mathrm{Gap}}-E_{\mathrm{NN}_{i}^{-}}$.

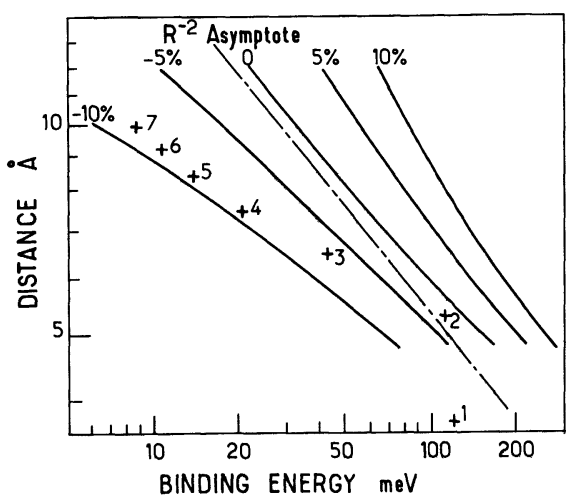

Fig. 1. - Log-Log plot of the pair separation $R$ versus the binding energy of $\mathrm{NN}^{-}$for different values of $\varepsilon$ $(-10 \%,-5 \%, 0,5 \%, 10 \%)$. The experimental points correspond to $\mathrm{NN}_{i}^{-}$in $\mathrm{GaP}(i=1$ to 7$)$.

In indirect gap semiconductors an accurate value of $E_{\mathrm{Gap}}$ is difficult to obtain. The data known accurately is the energy of the bottom of the free exciton band. Here, we have taken a value of the exciton Rydberg of $20 \mathrm{meV}$, the error being estimated to $\pm 2 \mathrm{meV}$. This error would affect to some extend the position of the points $i=5$ to 7 and the $\log -\log$ plot of figure 1 , but it would not affect the main conclusion : the experimental points at large $R(i=4$ to 7$)$ are clearly on the left of the asymptote. This shows unambiguously that $\varepsilon<0$ for $\mathrm{N}$ in $\mathrm{GaP}$. Also the slope at large $R$ is fairly well accounted for by our theory. The experimental slope approaching a $R^{-3}$ law [6] is 
also typical of a region $\varepsilon \sim-0.08$, and has nothing to do with a particular shape of the isoelectronic potential. From (12) one can evaluate the separation $R$ at which the binding of $\mathrm{NN}^{-}$ vanishes. One finds $R \sim 24 \AA(i \sim 40)$. Let us recall why the experimental data stop at $i=7$. This is because the $n S$ excited states are broadened when they are in resonance with the free exciton continuum.

In figure 1 , the theoretical curves were computed with a radius $b=2.4 \AA$. Changing $b$ would just move the theoretical curves parallel to the $R^{-2}$ asymptote. This corresponds to the fact that the relevant parameter of the $\mathrm{N}$ isoelectronic potential we can extract is $\varepsilon I_{A}$, which is about $0.04 \AA^{-1}$. For $E_{\mathrm{NN}^{-}}$larger than $40 \mathrm{meV}$, the model based on a simple parabolic band structure is no longer accurate. The deviation for $i=3,2$ is equivalent to an increase of $m^{*}$ for deeper levels. The very peculiar situation of $\mathrm{NN}_{1}$ could be related, we believe, to an overlap of the potentials of the two impurities, producing some non-linear effect. This is the only reason why we choose a value of $b$ such that $2 b$ is between the pair separation in $\mathrm{NN}_{1}$ and $\mathrm{NN}_{2}$. However, a smaller value of $b(\sim 1.5 \AA)$ is predicted in first principle theoretical calculation of isoelectronic potentials (2).

5. Conclusion. - We have shown that for an isoelectronic potential near the limit of vanishing binding, the study of pairs $\left(\mathrm{NN}_{i}\right)$ provides an unambiguous test for the stability of $\mathrm{N}^{-}$. This procedure is not sensitive to the exact shape of the electronic potential. On the other hand, one can try to deduce the stability of $\mathrm{N}^{-}$from the known binding energy of the exciton bound to $\mathrm{N}$. An attempt to do it through a simple Hartree self-consistent method has predicted a positive value of $\varepsilon$ [7]. This simply points out the necessity to include electron-hole correlation in such calculations.

Acknowledgments. - I thank P. Nozières for his encouragement and critics.

Appendix. - To deduce formally (4) from (3), we have to compute the overlap integral $D$. We assume that, at large $R$ and small $\eta$, the main part comes from the region outside the potentials. Then from (6)

$$
D=\frac{k}{2 \Pi} \int \mathrm{d} \tau \frac{\mathrm{e}^{-k\left(r_{1}+r_{2}\right)}}{r_{1} r_{2}} .
$$

The volume element is $\mathrm{d} \tau=\frac{r_{1} r_{2}}{R} \mathrm{~d} r_{1} \mathrm{~d} r_{2} \mathrm{~d} \varphi$ and in the quadrant $r_{1} r_{2}$ the domain of integration is limited by $r_{1}+r_{2}>R$ and $\left|r_{1}-r_{2}\right|<R$

$$
D=\frac{k}{R} \int \mathrm{d} r_{1} \mathrm{~d} r_{2} \mathrm{e}^{-k\left(r_{1}+r_{2}\right)}
$$

Using $r_{1}+r_{2}=x$ and $r_{1}-r_{2}=y$

$$
D=\frac{k}{2 R} \int_{-R}^{R} \mathrm{~d} y \int_{R}^{\infty} \mathrm{d} x \mathrm{e}^{-k x}=\mathrm{e}^{-k R} .
$$

Retaining the main significant terms in (3) we obtain :

$$
E_{1}=E(\eta)+\frac{B-\eta A}{1+D}
$$

Using (7), (13) and the definition of $k$

$$
E_{1}=-k^{2}+\frac{2 k}{R} \frac{k R-\exp (-k R)}{1+\exp (-k R)}
$$


Differentiation with respect to $k$ gives

$$
[k R-\exp (-k R)] \frac{1+\exp (-k R)+k R \exp (-k R)}{[1+\exp (-k R)]^{2}}=0,
$$

which is equivalent to (9).

\section{References}

[1] For a review, see e.g. CzaJA, W., Festkörperprobleme 11 (1971) 65.

[2] For a discussion on isoelectronic potentials, see BALDERESCHI, A., J. Lumin. 7 (1973) 79.

[3] Cohen, E. and Sturge, M. D., Phys. Rev. B 15 (1977) 1309.

[4] Thuselt, F., Kreher, K. and WüNSChe, H. J., Solid State Commun. 36 (1980) 563.

[5] Landau, L. and Lifchitz, E., Mécanique Quantique (ed. Mir. Moscow) 1967, p. 143.

[6] Allen, J. W., J. Phys. C 1 (1969) 1136.

[7] Benoît À la Guillaume, C., 16th Int. Conf. on the Physics of Semiconductors, Montpellier 1982. To be published by North Holland. 